Rev. Chil, Pediatr. 68 (5); 220-222, 1997

\title{
Arco para ventiladores mecánicos neonatales
}

\author{
Pablo Valenzuela F. ${ }^{1}$; Kerstins Arce J. ${ }^{2}$
}

\begin{abstract}
Resumen
La ventilacićn mecón ca pia-tea prodemas lécniccs en la fífación de las mengueras del ventiador y cel tubo endoiraqued. Para ese efecto se muestro un dissositivo ce acrilico en forma de arco de angu'ación varıble que satistace las necesidades de fijación de las mangueras y lubo endotraqueal. fócil menejo, aseo y adecuado acceso al recién nacido. El dispositivo éj además de bajo cosic y su usc tra sido frobado salisfacioriamente con el personal médico y de apoyo de $k$ unidad de rezín nacidos.
\end{abstract}

(Palabras clave ventiiación mecóniça: recién nacidos.]

\section{An acrilic arc to support mechanical ventilation hoses in newborn patients}

Appropriate fixing and manpulation of hoses and endaraceal iubes for mechanical ventilation poses several rechnica proslems fasicularily on small newoorn inlants. An asrilic sernicircular arc lined up by eight perpendicutar rods of the same material onc suppoted by ar articulated base which allows is place i: at different angles was designec and tesred. This low cost device a lows gcod fixat on of hases iogether with lair degres of freedon lor head mobilization, ready access for managment of er:doiracheal lukes, easies loilet of patients and their airway even inside siandard incutators and it has been rovoursoly evaluated by riurses and mecical personnel.

¡Key words: ventilatio-, mechanical, newborn.'

La ventilación mecánica es una de las eventualidades a las que frecuentemente está expuesto un recién nacido $(\mathrm{RN})$, especialmente los de muy bajo peso de nacimiento.

La interfase entre la máquina y el niño generalmente consiste en un sistema de mangueras flexibles que llegan a una pieza sobre la cual se conecta el tubo que se introducirá en Ja vía aérea (TET), sea por vía nasal u oral.

Esta interlase debe ser sustentada por algún dispositivo que permita la posición del TET desde cualquier punto, manteniendo una buena posición con respecto al recién nacido.

1. Unidad de Neonalología, Hospital Clínico L'niversidad de Chile.

2. Matrona. Unidad de Neonatología, Hospital Clínico Universidud de Chile.

Equipo fabricado con la colaboración de Procom Ltda., Santiago. Chile.
Para la solución de estos problemas se han desarrollado diversos dispositivos de soporte $y$ contención de las mangueras del ventilador, todos los cuales son de difícil obtención en nuestro medio. Frente a la dificultad de conseguir un dispositivo de esta naturaleza en nuestro mercado, nos propusimos desarrollar uno que cumpliera dichos propósitos y que fuera bien aceptado por el personal encargado de la atención de los recién nacidos.

\section{Material y Método}

Se construyó un aparato (patente en trámite) en forma de arco. en acrílico transparente, radio lúcido, que consta de un pie $o$ base en forma de semicírculo articulado en cada uno de sus extremos inferiores. La altura del arco deja un espacio adecuado para la cabeza del niño y al misno tiempo permite su empleo dentro de una incubadora estándar. La articulación de la base puede fijarse con tornillos de mariposa en tres posiciones: $45^{\circ}$ hacia atrás, adelante o vertical. El sistema de mariposas permite también 




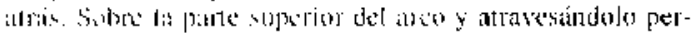

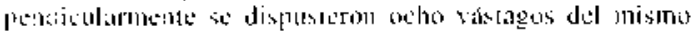

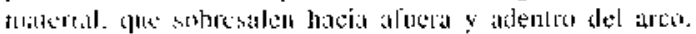

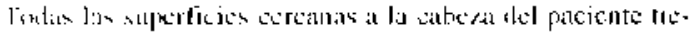

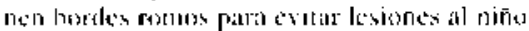

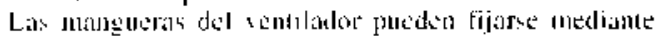

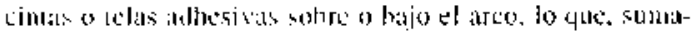

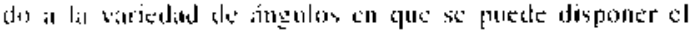
aren, permatc la apoximasion al la cabera del rectén naci-

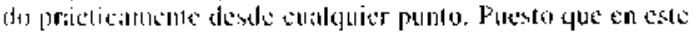
supore so fijan las mangueras de conexibul y no el tubu

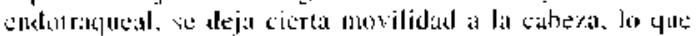


Y 2 .

\section{Resultados}

Dexide el monento de su labricación, cada ver que ha sido nocesarion. se ha utiliźdu el arco demostrindese cliciente en cuanto a la sujeción del tuho. permitir un adecuado aceeso a



tubo en el momento de las aspiraciones, hacer posible un buen asco y no interferit con la radiolog̣ia. Por no tener aún un grupo control no hemos podido medir cuantitativamente sus venlajas en cuanto a descanulaciones accidentales 0 malas posiciones del tubo endotraqueal. pero entre el personal existe una impresión favorable gencralizada hacia el dispositivo.

\section{Comentario}

Los efectos que estos dispositivos tienen sobre el niño han sido estudiados desde que esta técnica se hizo habitual en el año 1960'. Hay diversos problemas derivados de la estabilidad del tubo endotraqueal dentro de la vía aérea y de la interacción entre aquel y las paredes de esta.

Las mangueras y tubos de conexion ticnen peso y flexibilidad propias, con algunas partes

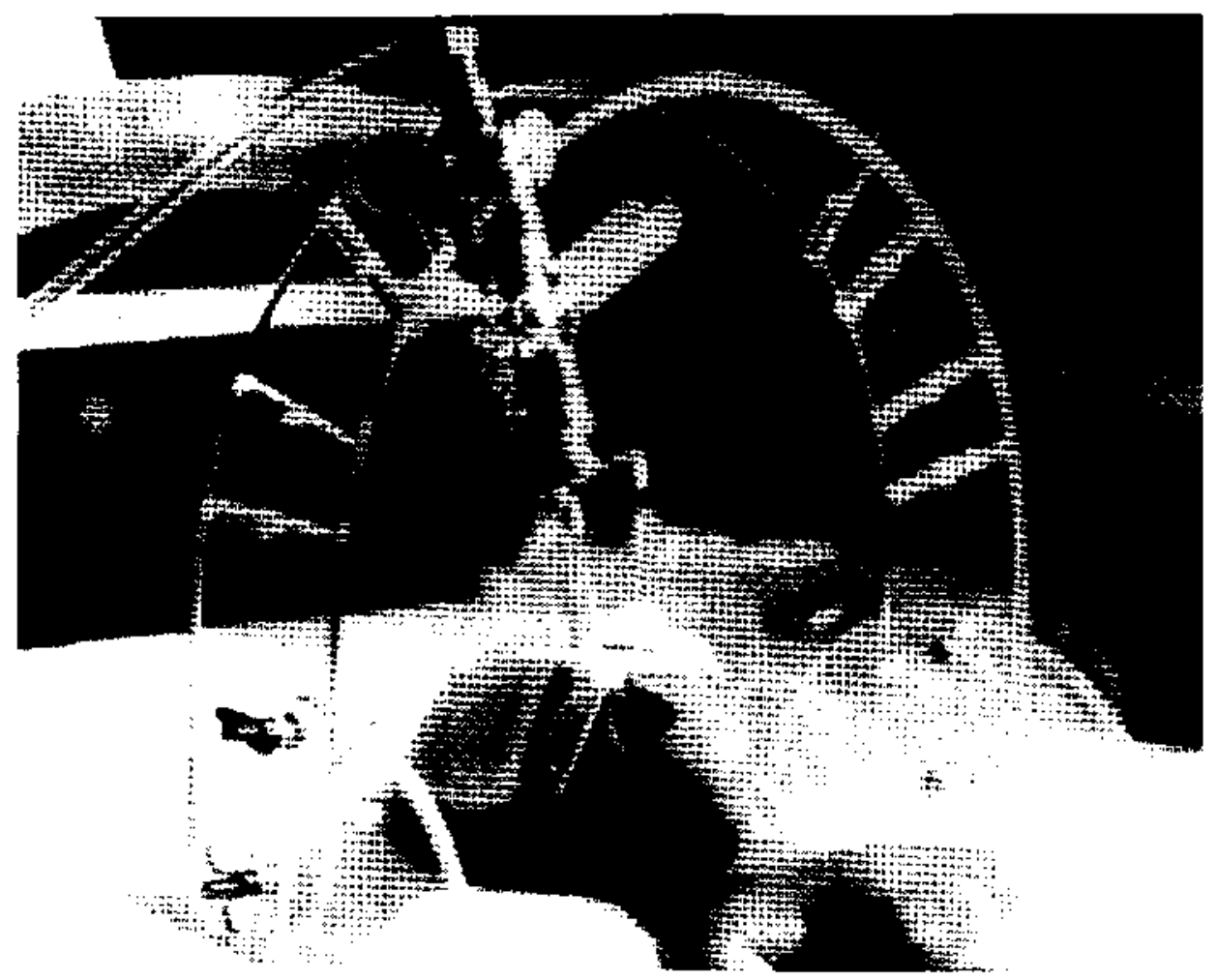

Higura I: Vista gencial del arco durante la ventilación mecánica de un recién nacido pretérmino de

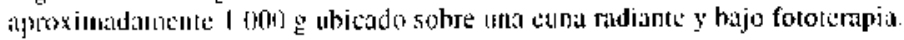




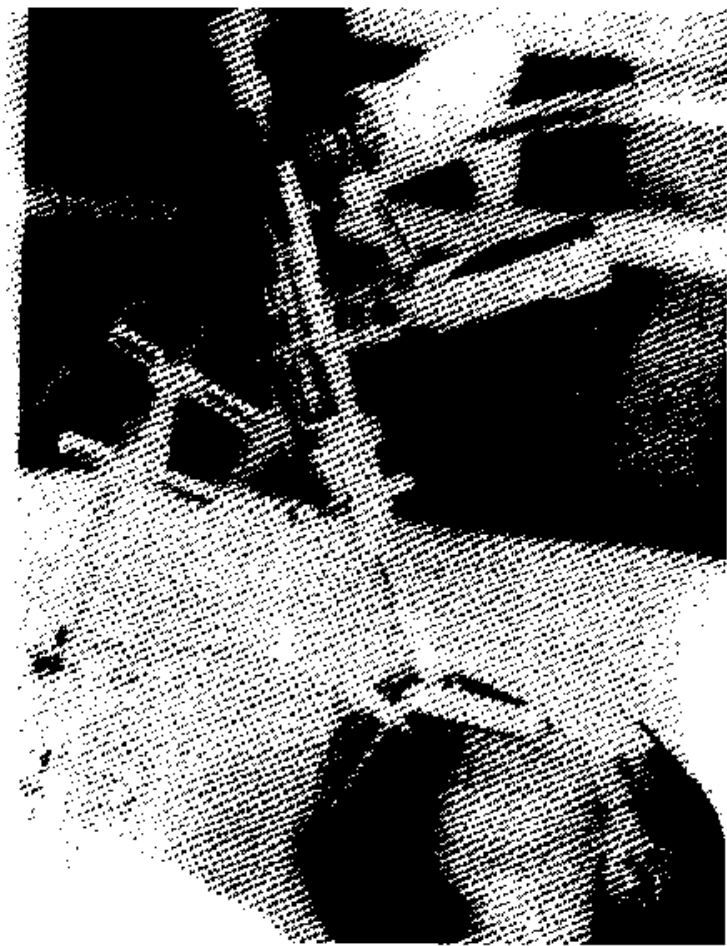

Figura 2: Vista de aproximación del áco para ver el modu e que las mangueras etián sujetas al arco. glue en esto caso se realiza por el solo efecto de los váslagos, sin tclas adlexiviss ni cintás dando gran uperabilidad al sistemia

may lígidas, que genelan fucrzas que se transmiten hasta el tubo endotraqueal, que habitualmente es muy flexible, obligándolo a tomar posiciones que pueden ser perjudiciales para el neonato, pues tienden a introducirse demasiado, acolarse o salirse fácilmente.

Los movimientes de la cabeza del recién nacido desplazan al tubo traqueal en torma signilicativila ${ }^{2}, i$ y el roce en los lugares de entrada puede producir serias lesiones, especialmente en las narinas ${ }^{+h}$. In laringe y la traquea ${ }^{7,8}$. Por otra palte, en cada respiración se producen pequeños movimientos de todo el sistema de mangueras, los que pueden transmitirse al dicho tubo con el contiguiente daño de los lejidos sobre los cuales se apoya.

Muchas veces las mangueras del ventilador y la conexión al tubo son más voluminosos que la cabcza del recién nacido, haciendo muy difícil la cstahilidad de la conexión. Súmese a todo esto el manejo dentro de una incubadora y se tendrá un cuadro aproximado de las difícultades con que se desarrollan los cuidados de enfermería en casos de ventilación mecánica neonatal.

Nucsiro propósito fue construir un aparato que permitiera un adccuado manejo del problema, posible de fabricar en el país, barato, fácil de ascar y compatible con los ventiladores en uso y las incubadoras neonatales. El aparato diseñado cumple satisfactoriamente los propósitos para los que fue fabricado con un adecuado grado de accptación tanto entre las matronas como entre los médicos, es fácil de opcrar, permite una fácil fijación de las mangueras dé] ventilador y del tubo traqueal, no es traumático, es barato y por to tanto aplicable en unidades de neonatología que cuentan con los recursos que son habituales en nuestro medio.

\section{Agradecimientos}

Agladecemos a Procom Lida por la ayuda en la fabricación del areo y muy especialmente al Sr. Ronald Phillips $V$., puiss sin sa ayuda clia habría sido muy difícil.

\section{Referencias}

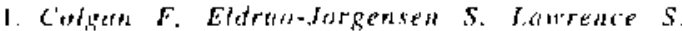
Mainsenance of respiration in the neonalal respiralory distress syndroune. JAMA 1960: 173: 1557-1559,

2. Fodre's $D$, de Bros $F$, Krames $S$; Endotracheal tubs displacencont in the newborn infant. J Pcdiatr 1976: 89 : $126-127$

3. Down $S$. Bleric C: Endotracheal tube movement in the preterm neonate: oral versus basal intubation. Anח Ocol Rhinol Laryngol 1985; 94: 18-20.

4. Jang A. Themas 6 : Stricture of the nasal vestibule: a complacation of nasotrachial intubation in newbom intants. J Pedialt 1974; 85: 412-414

5. Commer $f$, Buthty $M$, Matsels $M$ : Prolongued orotracheal intubation in the newhorn. Ann Onl 1980: 89: $459-46$ ]

6. Rivera $R$. Tibhedts S, Ftaracs MB: Complications of endotracheal intubation and mechanical ventilation in inlinls and childten. Cri Care Med 1992: 20: J43-199

7. Shermeth J. Nelson $H$ : Decreased incidence o[ subglocic itenosis using an approprialed-sized endotracheal tube in neonates. Pulmonology 1989; 6: 183-185.

8. Morrissey M, Bailer C: Didgnosis and management of subrylotiti stinosic after neonatal ventilation. Arch Dis Child [590; $65 ; 110)^{3}-1104$ 